\title{
G-STRUCTURES OF SECOND ORDER DEFINED BY LINEAR OPERATORS SATISFYING ALGEBRAIC RELATIONS
}

\author{
Demetra Demetropoulou-Psomopoulou
}

\begin{abstract}
The present work is based on a type of structures on a differential manifold $V$, called $G$-structures of the second kind, defined by endomorphism $J$ on the second order tangent bundle $T^{2}(V)$. Our objective is to give conditions for a differential manifold to admit a real almost product and a generalised almost tangent structure of second order. The concepts of the second order frame bundle $H^{2}(V)$, its structural group $L^{2}$ and its associated tangent bundle of second order $T^{2}(V)$ of a differentiable manifold $V$, are used from the point of view that is described in papers [5] and [6]. Also, the almost tangent structure of order two is mentioned and its generalisation, the second order almost transverse structure, is defined.
\end{abstract}

\section{Introduction}

A special type of first kind $G$-structures on an $n$-dimensional differentiable manifold $V_{n}$, are those defined by a differentiable field of linear operators $J_{x}$, such that at each point $x \in V_{n}, J_{x}$ maps the tangent space $T_{x}\left(V_{n}\right)$ into itself and satisfies algebraic relations.

Thus if $J_{x}^{2}=0$ and $J_{x}$ is of rank $p(2 p \leq n)$ everywhere, then there is defined a $G_{1}$-structure $([\mathbf{1 2}],[\mathbf{1 4}])$, the so called generalised almost tangent structure $([\mathbf{2}])$. In particular, if $n=2 p$, then the manifold $V_{n}$ has an almost tangent structure, briefly (a.t.)-structure ([4], [11]).

If $J^{2} x=I$, then there is, defined on $V_{n}$, a real almost product structure, briefly $\pi_{R}$-structure, of dimension $\left(n_{1}, n_{2}\right)$ with $n_{1}+n_{2}=n([\mathbf{1 3}])$.

A generalisation to the second order of the $\pi_{R}$-structure and the $G_{1}$-structure, respectively $\pi_{R}^{2}$-structure and $G_{1}^{2}$-structure, is given in [5] and [6], by means of a differential field of linear operators $J_{x}$, acting on the space $T_{x}^{2}\left(V_{n}\right)$ of the second order tangent vectors and satisfying the same algebraic equations. 
For this purpose the concepts of the frame bundle and tangent bundle of second order are given with the use of the jet theory discussed in works of C. Ehresmann ([7], [8], [9], [10]). The notions of the second order tangent vector coincides with that defined by $[\mathbf{3}]$. However there must be noticed that there are other, different definitions of the tangent bundle of higher order, in general ([15], [17]).

From the same standpoint in the present paper, there is given on $V_{n}$, at first, a $(\text { a.p. })_{R}^{2}$-structure of dimension

$$
\left(n+\left(\begin{array}{c}
n+1 \\
2
\end{array}\right)-\left(p+\left(\begin{array}{c}
p+1 \\
2
\end{array}\right)\right), p+\left(\begin{array}{c}
p+1 \\
2
\end{array}\right)\right)
$$

(that is an almost product structure of second order), induced from an $G_{1}^{2}$-structure of rank $p+\left(\begin{array}{c}p+1 \\ 2\end{array}\right)$.

Additionally, the second order almost tangent structure, briefly (a.t. $)^{2}$ structure, a special case of the $G_{1}^{2}$-structure $([\mathbf{6}])$, is mentioned. This structure is a particular case of the $(a . t r .)^{2}$-structure too, that is also defined, and is a generalisation to the second order of the almost transverse structure $([\mathbf{1 6}])$.

Finally, with the help of the previous structures, to a $G_{1}^{2}$-structure of rank $p+\left(\begin{array}{c}p+1 \\ 2\end{array}\right)$, there is given a compatible $(a . p .)_{R}^{2}$-structure of dimension

$$
\left(n+\left(\begin{array}{c}
n+1 \\
2
\end{array}\right)-2\left(p+\left(\begin{array}{c}
p+1 \\
2
\end{array}\right)\right), 2\left(p+\left(\begin{array}{c}
p+1 \\
2
\end{array}\right)\right)\right) .
$$

\section{Preliminaries}

We recall the following, from the concepts given by C. Ehresmann ([7], $[\mathbf{8}],[\mathbf{9}],[\mathbf{1 0}])$ and used in $[\mathbf{5}]$ and $[\mathbf{6}]$ :

Let $V_{n}$ be an $n$-dimensional differentiable manifold of class $C^{\infty}$. The second order frame bundle $H^{2}\left(V_{n}\right)=\bigcup_{x \in V_{n}} H_{x}^{2}\left(V_{n}\right)$ is a principal fibre bundle with basis $V_{n}$ and structural group $L_{n}^{2}$, where $H_{x}^{2}$ is the set of all invertible 2-jets of $R^{n}$ into $V_{n}$ with source $0 \in R^{n}$ and target $x \in V_{n}$. It can be identified ([5]) with the space of bases of the vector space $T_{x}^{2}\left(V_{n}\right)$, at $x \in V_{n}$, of the second order tangent vectors, as those are defined by $[3]$.

The Lie group $L_{n}^{2}$, that is the set $j_{0}^{2} f$ of all invertible 2-jets with source and target $0 \in R^{n}$ of a 2-mapping $f$ at the point $0 \in R^{n}$, can be identified 
([5], [6]), with a subgroup of matrices $G l(N, R)$, where $N=n+\frac{n(n+1)}{2}$. To the element $a \in L_{n}^{2}$,

$$
\begin{array}{r}
a=\left(a_{j_{1}}^{i}, a_{j_{1} j_{2}}^{i}\right), \quad i, j_{1}, j_{2}=1,2, \ldots n, \operatorname{det}\left(a_{j}^{i}\right) \neq 0 \text { and } a_{j_{1} j_{2}}^{i} \\
\text { symmetric with respect to } j_{1}, j_{2},
\end{array}
$$

corresponds, the matrix $A$ of the form,

$$
A=\left[\begin{array}{cc}
a_{j_{1}}^{i} & 0 \\
a_{j_{1} j_{2}}^{i} & a_{j_{1}}^{i_{1}} a_{j_{2}}^{i_{2}}
\end{array}\right] .
$$

We have, $\operatorname{dim} L_{n}^{2}=n^{2}+n\left(\begin{array}{c}n+1 \\ 2\end{array}\right)$.

The tangent bundle of the second order, $T^{2}\left(V_{n}\right)=\bigcup_{x \in V_{n}} T_{x}^{2}\left(V_{n}\right)$ has ([5], [6]) basis $V_{n}$, structural group $L_{n}^{2}$ and fibre $F^{2}=\left(L_{1, n}^{2}\right)^{*}$, where $L_{1, n}^{2}$ is the set $j_{0}^{2} g$ of all 2-jets with source $0 \in R^{n}$ and target $0 \in R$ of a 2-mapping $g$ at $0 \in R^{n}$. This bundle is $([\mathbf{5}])$ the dual vector bundle of $T_{1}^{2 *}\left(V_{n}\right)=\bigcup_{x \in V_{n}} T_{1, x}^{2 *}\left(V_{n}\right)$, with basis $V_{n}$, structural group $L_{n}^{2}$ and fibre $L_{1, n}^{2}$.

Each element $\omega$ of the vector space $L_{1, n}^{2}$, isomorphic to the fibre $T_{x}^{2 *}\left(V_{n}\right)=T_{1, x}^{2 *}\left(V_{n}\right)$, can be written in the form,

$$
\omega=\left[\begin{array}{c}
\omega_{i_{1}} \\
\omega_{i_{1} i_{2}}
\end{array}\right], \quad i_{1}, i_{2}=1,2, \ldots, n \text { and } \omega_{i_{1} i_{2}}
$$

symmetric with respect to the indices $i_{1}, i_{2}$.

Also, $\operatorname{dim} L_{1, n}^{2}=\operatorname{dim} T_{x}^{2 *}\left(V_{n}\right)=n+\left(\begin{array}{c}n+1 \\ 2\end{array}\right)$.

For two given charts, the transformation law for the coordinates of an element of $T^{2 *}\left(V_{n}\right)$, is given by the form,

$$
\begin{aligned}
x^{i^{\prime}} & =x^{i^{\prime}}\left(x^{j}\right), \\
{\left[\begin{array}{c}
\omega_{j_{1}^{\prime}} \\
\omega_{j_{1}^{\prime} j_{2}^{\prime}}^{\prime}
\end{array}\right] } & =\left[\begin{array}{cc}
a_{j_{1}^{\prime}}^{i_{1}} & 0 \\
a_{j_{1}^{\prime} j_{2}^{\prime}}^{i_{1}} & a_{j_{1}^{\prime}}^{i_{1}} \\
i_{j_{2}^{\prime}}
\end{array}\right]\left[\begin{array}{c}
\omega_{i_{1}} \\
\omega_{i_{1} i_{2}}
\end{array}\right],
\end{aligned}
$$

where

$$
\left(a_{j_{1}^{\prime}}^{i_{1}}=\frac{\partial x^{i_{1}}}{\partial x^{j_{1}^{\prime}}}, a_{j_{1}^{\prime} j_{2}^{\prime}}^{i_{1}}=\frac{\partial^{2} x^{i_{1}}}{\partial x^{j_{1}^{\prime}} \partial x^{j_{2}^{\prime}}}\right) \in L_{n}^{2} \text { and }\left\{x^{i}\right\}_{i=1,2, \ldots, n},\left\{x^{j^{\prime}}\right\}_{j=1,2, \ldots, n}
$$


are two systems of local coordinates at $x \in V_{n}$.

Let the $n+\left(\begin{array}{c}n+1 \\ 2\end{array}\right)$ second order tangent vectors,

$$
\left(e_{i_{1}}, e_{i_{1} i_{2}}\right), \quad i_{1}, i_{2}=1,2, \ldots, n \text { and } e_{i_{1} i_{2}}
$$

symmetric in the indices $i_{1}, i_{2}$,

define the natural basis for $T_{x}^{2}$, for the local chart $\left\{x^{i}\right\}_{i=1,2, \ldots, n}$ at the point $x \in V_{n}$. Then, every $v \in T_{x}^{2}$ can be expressed uniquely in the form,

$$
v=\left[v^{i_{1}} v^{i_{1} i_{2}}\right], \quad i_{1}, i_{2}=1,2, \ldots, n \text { and } v^{i_{1} i_{2}}
$$

symmetric in the indices $i_{1}, i_{2}$.

For another system of local coordinates $\left\{x^{j^{\prime}}\right\}_{j=1,2, \ldots, n}$ at $x \in V_{n}$ the second order tangent vectors $\left(e_{j_{1}^{\prime}}, e_{j_{1}^{\prime} j_{2}^{\prime}}\right)$ of a new basis of $T_{x}^{2}$, are transformed to the basis (1.4) by the matrices,

and

$$
\left[\begin{array}{c}
e_{i_{1}} \\
e_{i_{1} i_{2}}
\end{array}\right]=\left[\begin{array}{cc}
a_{i_{1}}^{j_{1}^{\prime}} & 0 \\
a_{i_{1} i_{2}}^{j_{1}^{\prime}} & a_{i_{1}}^{j_{1}^{\prime}} a_{i_{2}}^{j_{2}^{\prime}}
\end{array}\right]\left[\begin{array}{c}
e_{j_{1}^{\prime}} \\
e_{j_{1}^{\prime} j_{2}^{\prime}}
\end{array}\right],
$$

$$
\left[\begin{array}{cc}
a_{i_{1}}^{j_{1}^{\prime}} & 0 \\
a_{i_{1} i_{2}}^{j_{1}^{\prime}} & a_{i_{1}}^{j_{1}^{\prime}} a_{i_{2}}^{j_{2}^{\prime}}
\end{array}\right]
$$

is the corresponding matrix to the element $\left(a_{i_{1}}^{j_{1}^{\prime}}, a_{i_{1} i_{2}}^{j_{1}^{\prime}}\right) \in L_{n}^{2}$ (inverse of that given in the relations (1.3)). The transformation law for the local coordinates of $v \in T_{x}^{2}$ is given by the matrices,

$$
\left[\begin{array}{ll}
v^{j_{1}^{\prime}} & v^{j_{1}^{\prime} j_{2}^{\prime}}
\end{array}\right]=\left[\begin{array}{ll}
v^{i_{1}} & v^{i_{1} i_{2}}
\end{array}\right]\left[\begin{array}{cc}
a_{i_{1}}^{j_{1}^{\prime}} & 0 \\
a_{i_{1} i_{2}}^{j_{1}^{\prime}} & a_{i_{1}}^{j_{1}^{\prime}} a_{i_{2}}^{j_{2}^{\prime}}
\end{array}\right] .
$$

If $J_{x}$ is a differentiable field of linear operators acting on the space $T_{x}^{2}$, then the corresponding element $F$ of $T_{x}^{2} \otimes T_{x}^{2 *}$ can be represented by the matrix,

$$
\begin{aligned}
& F= {\left[\begin{array}{cc}
F_{i_{1}}^{j_{1}} & F_{i_{1}}^{j_{1} j_{2}} \\
F_{i_{1} i_{2}}^{j_{1}} & F_{i_{1} i_{2}}^{j_{1} j_{2}}
\end{array}\right], \quad i_{1}, i_{2}, j_{1}, j_{2}=1,2, \ldots, n, } \\
& F_{i_{1}}^{j_{1} j_{2}} \text { symmetric in } j_{1}, j_{2}, F_{i_{1} i_{2}}^{j_{1}} \text { symmetric in } i_{1}, i_{2} \\
& \text { and } F_{i_{1} i_{2}}^{j_{1} j_{2}} \text { symmetric in } i_{1}, i_{2} \text { and } j_{1}, j_{2} .
\end{aligned}
$$

It is defined by the relations,

$$
\left\{\begin{array}{l}
\left(J_{x} v\right)^{j_{1}}=F_{i_{1}}^{j_{1}} v^{i_{1}}+F_{i_{1} i_{2}}^{j_{1}} v^{i_{1} i_{2}}, \\
\left(J_{x} v\right)^{j_{1} j_{2}}=F_{i_{1}}^{j_{1} j_{2}} v^{i_{1}}+F_{i_{1} i_{2}}^{j_{1} j_{2}} v^{i_{1} i_{2}},
\end{array}\right.
$$

where $v=\left(v^{i_{1}}, v^{i_{1} i_{2}}\right)$, is a 2-tangent vector at $x \in V_{n}$. 


\section{A $(a . p .)_{R}^{2}$-structure induced from a $G_{1}^{2}$-structure}

I. A real almost product structure of second order is defined ([5] on an $n$-dimensional differentiable manifold $V_{n}$ of class $C^{\infty}$, by a linear operator $J_{x}$ acting on the space $T_{x}^{2}\left(V_{n}\right)$ of the second order tangent vectors at each point $x \in V_{n}$ and satisfying the equation

$$
J_{x}^{2}=I
$$

Then $J_{x}$ gives a decomposition of $T_{x}^{2}$ in a direct sum of two complementary subspaces.

If we assume that,

(i) $L$ and $M$ are the two proper supplementary subspaces of $T_{x}^{2}$ corresponding to the eigenvalues -1 and +1 respectively, according to the relation $(2.1)$,

(ii) $\operatorname{dim} L=n+\left(\begin{array}{c}n+1 \\ 2\end{array}\right)-\left(p+\left(\begin{array}{c}p+1 \\ 2\end{array}\right)\right)=q+\left(\begin{array}{c}q+1 \\ 2\end{array}\right)+q p$, $q=n-p, \operatorname{dim} M=p+\left(\begin{array}{c}p+1 \\ 2\end{array}\right)$, then, there is defined on $V_{n}$ an (a.p. $)_{R}^{2}$-structure of dimension $\left(q+\left(\begin{array}{c}q+1 \\ 2\end{array}\right)+q p, p+\left(\begin{array}{c}p+1 \\ 2\end{array}\right)\right)$, with $n-p=q$. It is, also a generalisation of the real almost

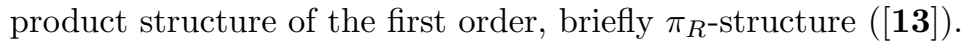

Remark. It must be noticed that in [5] there is discussed a real almost product structure of second order, the $\pi_{R}^{2}$-structure, with different dimension.

Its adapted basis can be defined by

$$
\begin{aligned}
& \left\{\left(e_{\alpha_{1}}, e_{A_{1}}\right)\left(e_{\alpha_{1} \alpha_{2}}, e_{\alpha_{1} A_{2}}, e_{A_{1} A_{2}}\right),\right. \\
& \quad \alpha_{1}, \alpha_{2}=1,2, \ldots, q, A_{1}, A_{2}=q+1,2, \ldots, q+p=n,
\end{aligned}
$$

where the 2-tangent vectors,

$$
\left\{e_{\alpha_{1}}, e_{\alpha_{1} \alpha_{2}}, e_{\alpha_{1} A_{2}}\right\} \text { and }\left\{e_{A_{1}}, e_{A_{1} A_{2}}\right\}
$$

form a basis of $L$ and $M$ respectively. 
The matrix $A$ of the transformation for the adapted bases is of the form,

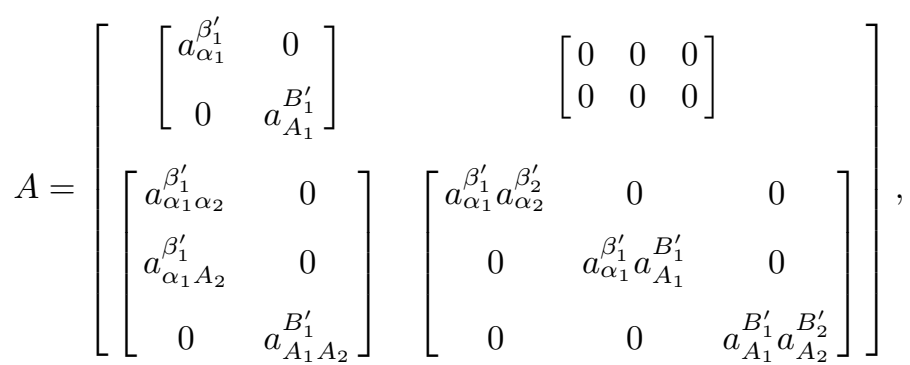

with

$$
\left[\begin{array}{cc}
a_{\alpha_{1}}^{\beta_{1}^{\prime}} & 0 \\
0 & a_{A_{1}}^{B_{1}^{\prime}}
\end{array}\right] \in L_{(n-p, p)}, a_{\alpha_{1}}^{\beta_{1}^{\prime}} \in L_{q}, a_{A_{1}}^{B_{1}^{\prime}} \in L_{p},
$$

where $L_{(n-p, p)}$ is $([\mathbf{1 3}])$ the structural group of the $\pi_{R}$-structure.

Thus, this $(a . p .)_{R}^{2}$-structure is a $G$-structure of second order whose structural group $L_{(n-p, p)}^{2}$ is consisting of matrices of the form (2.2), subgroup of $L_{n}^{2}$.

The corresponding tensor $F$ to the operator $J$ for the $(a . p .)_{R}^{2}$-adapted basis can be represented by the matrix,

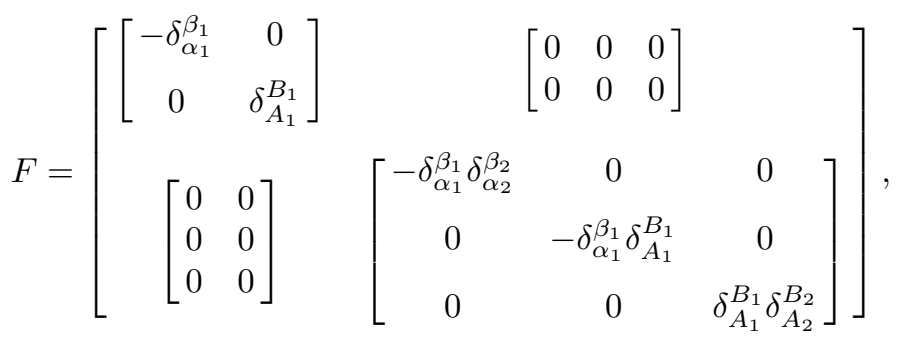

and we have

$$
F A=A F
$$

for every element $A$ of the structural group $L_{(n-p, p)}^{2}$ of the $(a . p .)_{R^{-}}^{2}$ structure.

II. An $n$ dimensional differentiable manifold $V_{n}$ admits ([6]) a generalised almost tangent structure of second order, briefly $G_{1}^{2}$-structure, if there exists a differentiable field of linear operators $J_{x}$ of constant rank $p+\left(\begin{array}{c}p+1 \\ 2\end{array}\right)$, such that at each point $x \in V_{n}$,

$$
\left\{\begin{array}{l}
J_{x} \text { maps } T_{x}^{2}\left(V_{n}\right) \text { into itself } \\
J_{x}^{2}=0
\end{array}\right.
$$


The differentiable manifold $V_{n}$ is called $G_{1}^{2}$-manifold, and

$$
n+\left(\begin{array}{c}
n+1 \\
n
\end{array}\right)>2\left(p+\left(\begin{array}{c}
p+1 \\
2
\end{array}\right)\right), \quad n \geq 2 p
$$

Let

$$
\begin{array}{r}
\left\{\left(e_{\alpha_{1}(1)}, e_{\alpha_{1}}, e_{\alpha_{1}(2)}\right),\left(e_{\alpha_{1}(1) \alpha_{2}(1)}, e_{\alpha_{1}(1) \alpha_{2}}, e_{\alpha_{1}(1) \alpha_{2}(2)},\right.\right. \\
\left.\left.e_{\alpha_{1} \alpha_{2}(2)}, e_{\alpha_{1} \alpha_{2}}, e_{\alpha_{1}(2) \alpha_{2}(2)}\right)\right\} \\
\alpha(1)=1,2, \ldots, p, \alpha=p+1, \ldots, q, \alpha(2)=q+1, \ldots, q+p=n
\end{array}
$$

be a basis of $T_{x}^{2}\left(V_{n}\right)$, such that the 2-tangent vectors

$$
\left\{\left(e_{\alpha_{1}(1)}, e_{\alpha_{1}}\right),\left(e_{\alpha_{1}(1) \alpha_{2}(1)}, e_{\alpha_{1}(1) \alpha_{2}}, e_{\alpha_{1}(1) \alpha_{2}(2)}, e_{\alpha_{1} \alpha_{2}}, e_{\alpha_{1} \alpha_{2}(2)}\right)\right\}
$$

form a basis of Ker $J_{x}$ and the tangent vectors of second order basis of $S_{x}$ (complement space of $\operatorname{Ker} J_{x}$ in $T_{x}^{2}(V)$ )

$$
\left\{e_{\alpha_{1}(2)}, e_{\alpha_{1}(2) \alpha_{2}(2)}\right\}
$$

satisfy two conditions,

$$
\left\{\begin{array}{l}
J_{x} e_{\alpha_{1}(2)}=e_{\alpha_{1}(1)} \\
J_{x} e_{\alpha_{1}(2) \alpha_{2}(2)}=e_{\alpha_{1}(1) \alpha_{2}(1)}
\end{array}\right.
$$

Such a basis is called ([6]) an $G_{1}^{2}$-adapted basis. The matrix $B$ of the transformation for the $G_{1}^{2}$-adapted bases is of the form,

$$
B=\left[\begin{array}{cc}
b_{i_{1}}^{j_{1}^{\prime}} & 0 \\
b_{i_{1} i_{2}}^{j_{1}^{\prime}} & b_{i_{1}}^{j_{1}^{\prime}} b_{i_{2}}^{j_{2}^{\prime}}
\end{array}\right]
$$

with

$$
b_{i_{1}}^{j_{1}^{\prime}}=\left[\begin{array}{ccc}
b_{\alpha_{1}(1)}^{\beta_{1}^{\prime}(1)} & 0 & 0 \\
b_{\alpha_{1}}^{\beta_{1}^{\prime}(1)} & b_{\alpha_{1}}^{\beta_{1}^{\prime}} & 0 \\
b_{\alpha_{1}(2)}^{\beta_{1}^{\prime}(1)} & b_{\alpha_{1}(2)}^{\beta_{1}^{\prime}} & b_{\alpha_{1}(1)}^{\beta_{1}^{\prime}(1)}
\end{array}\right] \in G_{1},
$$

$b_{\alpha_{1}(1)}^{\beta_{1}^{\prime}(1)}=b_{\alpha_{1}(2)}^{\beta_{1}^{\prime}(2)} \in L_{p}, b_{\alpha_{1}}^{\beta_{1}^{\prime}(1)} \in \operatorname{Hom}\left(R^{p}, R^{n-2 p}\right), b_{\alpha_{1}}^{\beta_{1}^{\prime}} \in L_{n-2 p}, b_{\alpha_{1}(2)}^{\beta_{1}^{\prime}(1)} \in$ $\operatorname{End}\left(R^{p}, R^{p}\right), b_{\alpha_{1}(2)}^{\beta_{1}^{\prime}} \in \operatorname{Hom}\left(R^{n-2 p}, R^{p}\right), G_{1}$ is the structural group of the 
$G_{1}$-structure $([\mathbf{2}],[\mathbf{1 2}],[\mathbf{1 4}])$, and

$$
b_{i_{1} i_{2}}^{j_{1}^{\prime}}=\left[\begin{array}{ccc}
b_{\alpha_{1}(1) \alpha_{2}(1)}^{\beta_{1}^{\prime}(1)} & 0 & 0 \\
b_{\alpha_{1}(1) \alpha_{2}}^{\beta_{1}^{\prime}(1)} & b_{\alpha_{1}(1) \alpha_{2}}^{\beta_{1}^{\prime}} & 0 \\
b_{\alpha_{1}(1) \alpha_{2}(2)}^{\beta_{1}^{\prime}(1)} & b_{\alpha_{1}(1) \alpha_{2}(2)}^{\beta_{1}^{\prime}} & 0 \\
b_{\alpha_{1} \alpha_{2}}^{\beta_{1}^{\prime}(1)} & b_{\alpha_{1} \alpha_{2}}^{\beta_{1}^{\prime}} & 0 \\
b_{\alpha_{1} \alpha_{2}(2)}^{\beta_{1}^{\prime}(1)} & b_{\alpha_{1} \alpha_{2}(2)}^{\beta_{1}^{\prime}} & 0 \\
b_{\alpha_{1}(2) \alpha_{2}(2)}^{\beta_{1}^{\prime}(1)} & b_{\alpha_{1}(2) \alpha_{2}(2)}^{\beta_{1}^{\prime}} & b_{\alpha_{1}(1) \alpha_{2}(1)}^{\beta_{1}^{\prime}(1)}
\end{array}\right],
$$

It can be noticed that the matrices,

$$
\left[\begin{array}{cc}
b_{\alpha_{1}(1)}^{\beta_{1}^{\prime}(1)} & 0 \\
b_{\alpha_{1}}^{\beta_{1}^{\prime}(1)} & b_{\alpha_{1}}^{\beta_{1}^{\prime}}
\end{array}\right] \text { and }\left[\begin{array}{cc}
b_{\alpha_{1}(1) \alpha_{2}(1)}^{\beta_{1}^{\prime}(1)} & 0 \\
b_{\alpha_{1}(1) \alpha_{2}}^{\beta_{1}^{\prime}(1)} & b_{\alpha_{1}(1) \alpha_{2}}^{\beta_{1}^{\prime}} \\
b_{\alpha_{1}(1) \alpha_{2}(2)}^{\beta_{1}^{\prime}(1)} & b_{\alpha_{1}(1) \alpha_{2}(2)}^{\beta_{1}^{\prime}} \\
b_{\alpha_{1} \alpha_{2}}^{\beta_{1}^{\prime}(1)} & b_{\alpha_{1} \alpha_{2}}^{\beta_{1}^{\prime}} \\
b_{\alpha_{1} \alpha_{2}(2)}^{\beta_{1}^{\prime}(1)} & b_{\alpha_{1} \alpha_{2}(2)}^{\beta_{1}^{\prime}}
\end{array}\right]
$$

from (2.7) and (2.8) respectively, express the basis transformation of Ker $J_{x}$.

Thus, a $G_{1}^{2}$-structure is a $G_{1}$-structure of the second order whose structural group $G_{1}^{2}$ is consisting of matrices of the form (2.6) (with (2.7) and (2.8)), subgroup of $L_{n}^{2}$.

The tensor $F$ for the $G_{1}^{2}$-adapted basis can be represented by the 
matrix

$$
H=\left[\begin{array}{c}
{\left[\begin{array}{ccc}
0 & 0 & 0 \\
0 & 0 & 0 \\
\delta_{\alpha_{1}(1)}^{\beta_{1}(1)} & 0 & 0
\end{array}\right]} \\
{\left[\begin{array}{lll}
0 & 0 & 0 \\
0 & 0 & 0 \\
0 & 0 & 0 \\
0 & 0 & 0 \\
0 & 0 & 0 \\
0 & 0 & 0
\end{array}\right] \quad\left[\begin{array}{cccccc}
0 & 0 & 0 & 0 & 0 & 0 \\
0 & 0 & 0 & 0 & 0 & 0 \\
0 & 0 & 0 & 0 & 0 & 0
\end{array}\right]} \\
0 \\
0
\end{array}\right.
$$

and we have

$$
H B=B H,
$$

for every element $B$ of the structural group of the $G_{1}^{2}$-structure.

On the other hand, we have $([\mathbf{2}])$, that a differentiable manifold $V_{n}$ admits a distribution of dimension $n-p=q$ of the tangent space $T_{x}\left(V_{n}\right)$, if and only if, $V_{n}$ admits a $G_{(n-p, p)}$-structure, with structural group $G_{(n-p, p)}$, consisting of matrices of the form,

$$
\begin{aligned}
& {\left[\begin{array}{cc}
c_{\alpha_{1}}^{\beta_{1}^{\prime}} & 0 \\
c_{A_{1}}^{\beta_{1}^{\prime}} & c_{A_{1}}^{B_{1}^{\prime}}
\end{array}\right], \quad c_{\alpha_{1}}^{\beta_{1}^{\prime}} \in L_{q}, c_{A_{1}}^{\beta_{1}^{\prime}} \in \operatorname{End}\left(R^{q}, R^{p}\right), c_{A_{1}}^{B_{1}^{\prime}} \in L_{p},} \\
& \alpha=1,2, \ldots, q, A=q+1, \ldots, q+p=n,
\end{aligned}
$$

subgroup of $L_{n}$.

Similarly, a differentiable manifold $V_{n}$ admits a distribution of dimension $n+\left(\begin{array}{c}n+1 \\ 2\end{array}\right)-\left(p+\left(\begin{array}{c}p+1 \\ 2\end{array}\right)\right)=q+\left(\begin{array}{c}q+1 \\ 2\end{array}\right)+q p$ of the second order tangent space $T_{x}^{2}\left(V_{n}\right)$, if and only if, $V_{n}$ admits a $G_{(n-p, p)^{-}}$ structure of second order, that is a $G_{(n-p, p)}^{2}$-structure, with structural group $G_{(n-p, p)}^{2}$, consisting of matrices of the form,

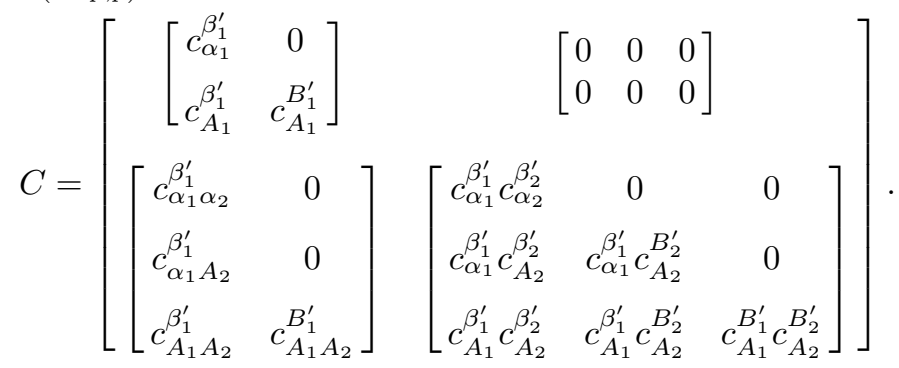


Obviously, the matrix $B\left((2.6)\right.$ with $(2.7)$ and (2.8)) of the group $G_{1}^{2}$ is a special case of the above matrix $C$. Therefore,

Proposition 2.1. If there is a given on the differentiable manifold $V_{n}$ a $G_{1}^{2}$-structure, there exist two distributions $L$ and $L_{1}$ of the second order tangent space $T_{x}^{2}\left(V_{n}\right)$, of dimension $n+\left(\begin{array}{c}n+1 \\ 2\end{array}\right)$ $\left(p+\left(\begin{array}{c}p+1 \\ 2\end{array}\right)\right)$ and $p+\left(\begin{array}{c}p+1 \\ 2\end{array}\right)$ respectively, such that $L_{1} \subseteq L$.

Proof: The spaces $L(x)=\operatorname{Ker} J_{x}$ and $L_{1}=J_{x}\left(S_{x}\right)$ satisfy the above conditions.

Thus, if the distribution $L$ of dimension $n+\left(\begin{array}{c}n+1 \\ 2\end{array}\right)-\left(p+\left(\begin{array}{c}p+1 \\ 2\end{array}\right)\right)$ is given, it is sufficient to define a distribution $M$ supplementary of $L$ in $T_{x}^{2}\left(V_{n}\right)$ of dimension $p+\left(\begin{array}{c}p+1 \\ 2\end{array}\right)$.

Consequently,

Proposition 2.2. If the differentiable manifold $V_{n}$ admits a $G_{1}^{2}$-structure, then the tensor field $F$ induces an $(a . p .)_{R}^{2}$-structure of dimension $\left(n+\left(\begin{array}{c}n+1 \\ 2\end{array}\right)-\left(p+\left(\begin{array}{c}p+1 \\ 2\end{array}\right)\right), p+\left(\begin{array}{c}p+1 \\ 2\end{array}\right)\right)$.

\section{3. (a.t. $)^{2}$ and (a.tr. $)^{2}$ structures}

I. The almost tangent structure of the first order, briefly (a.t.)-structure, is a particular case of the $G_{1}$-structure $([\mathbf{1 3}])$, if the differentiable manifold $V$ is of dimension $2 n$ and the rank of $J_{x}$ is equal to $n$. Then the group $G_{1}$ (relation (2.7)) reduces to the structural group $G\left({ }^{n}{ }_{n, n}\right)$ of the (a.t.)-structure $([\mathbf{4}],[\mathbf{1 1}])$, consisting of matrices of the form,

$$
\left[\begin{array}{cc}
K & 0 \\
N & K
\end{array}\right], \quad K \in L_{n}, N \in \operatorname{End}\left(R^{n}, R^{n}\right) .
$$

To the above structure there is a generalisation to the second order by means of a differentiable field of linear operators $J_{x}$, acting on the space $T_{x}^{2}\left(V_{2 n}\right)$ of the second order tangent vectors, with constant rank $n+\left(\begin{array}{c}n+1 \\ 2\end{array}\right)$. Obviously this second order almost tangent structure, briefly (a.t. $)^{2}$-structure, is a particular case of the $G_{1}^{2}$-structure, too. 
Thus, the components of the tensor $F$ are given by the matrix,

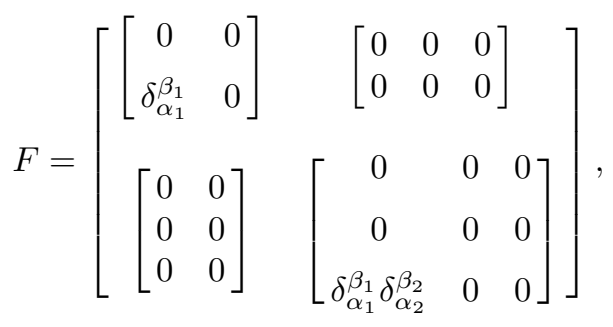

and the element $a=\left(a_{i_{1}}^{j_{1}^{\prime}}, a_{i_{1} i_{2}}^{j_{1}^{\prime}}\right) \in L_{2 n}^{2}$, by the matrix,

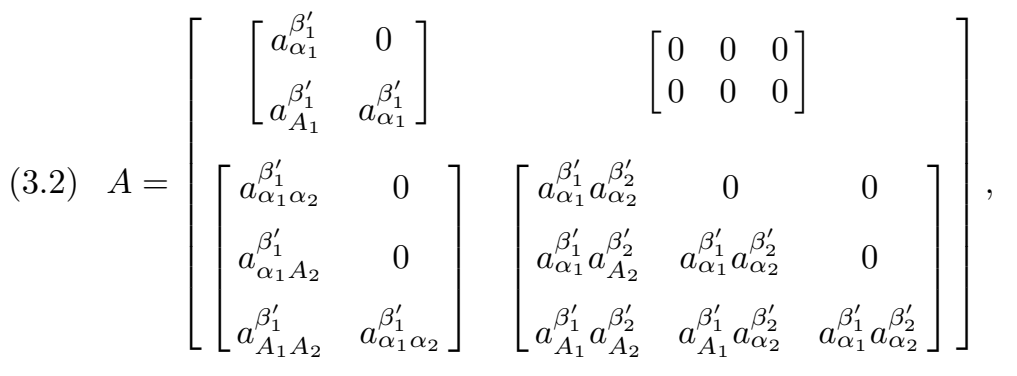

$$
\alpha_{1}, \alpha_{2}=1,2, \ldots, n, A_{1}, A_{2}=1,2, \ldots, 2 n \text { (that is } A=n+\alpha \text { ), }
$$

with

$$
\left[\begin{array}{cc}
a_{\alpha_{1}}^{\beta_{1}^{\prime}} & 0 \\
a_{A_{1}}^{\beta_{1}^{\prime}} & a_{\alpha_{1}}^{\beta_{1}^{\prime}}
\end{array}\right] \in G\left({ }_{n, n}^{n}\right), a_{\alpha_{1}}^{\beta_{1}^{\prime}}=a_{A_{1}}^{B_{1}^{\prime}} \in L_{n}, a_{A_{1}}^{\beta_{1}^{\prime}} \in \operatorname{End}\left(R^{n}, R^{n}\right) .
$$

We have,

$$
A F=F A
$$

for every element $A$ of the structural group $G^{2}\left({ }_{n, n}\right)$, of the $(a . t .)^{2}$ structure.

II. The notion of the almost transverse structure of the first order, briefly (a.tr.)-structure, is defined ([16]) on an $n$-dimensional manifold $V_{n}$ equipped with a foliation $\mathcal{L}$ of codimension $n-p$. That is, for an atlas $\left\{U, x^{\alpha}, x^{\alpha(1)}, x^{\alpha(2)}\right\}, \alpha=1,2, \ldots, n-p, \alpha(1), \alpha(2)=1,2, \ldots, p$, adapted to the foliation $\mathcal{L}$ the transformation functions verify the relation,

$$
\frac{\partial x^{\beta^{\prime}}}{\partial x^{\alpha(2)}}=0
$$


Then the space $L$ of the tangent vectors to the foliation $\mathcal{L}$, and the quotient space $Q=T\left(V_{n}\right) / L,(\operatorname{dim} Q=\operatorname{codim} L=n-p)$ define an (a.tr.)-structure with structural group formed by matrices:

$$
\begin{array}{r}
{\left[\begin{array}{ccc}
T & S & 0 \\
0 & K & 0 \\
0 & N & K
\end{array}\right], \quad T \in L_{n-p}, S \in \operatorname{Hom}\left(R^{p}, R^{n-p}\right)} \\
K \in L_{p}, N \in \operatorname{End}\left(R^{p}, R^{p}\right) .
\end{array}
$$

As it can be seen, this structure is a generalisation of the (a.t.)-structure, too.

Generalising the almost transverse structure to the second order, by adding the condition,

$$
\frac{\partial^{2} x^{\beta^{\prime}}}{\partial x^{\alpha_{1}(2)} \partial x^{\alpha_{2}(2)}}=0
$$

it can be defined an $(a . t r .)^{2}$-structure, whose structural group is of the form:

$$
\left[\begin{array}{cc}
l_{i_{1}}^{j_{1}^{\prime}} & 0 \\
l_{i_{1} i_{2}}^{j_{1}^{\prime}} & l_{i_{1}}^{j_{1}^{\prime}} l_{i_{2}}^{j_{2}^{\prime}}
\end{array}\right]
$$

with

$$
\begin{aligned}
l_{i_{1}}^{j_{1}^{\prime}}= & {\left[\begin{array}{ccc}
l_{\alpha_{1}}^{\beta_{1}^{\prime}} & l_{\alpha_{1}}^{\beta_{1}^{\prime}(1)} & 0 \\
0 & l_{\alpha_{1}(1)}^{\beta_{1}^{\prime}(1)} & 0 \\
0 & l_{\alpha_{1}(2)}^{\beta_{1}^{\prime}(1)} & l_{\alpha_{1}(1)}^{\beta_{1}^{\prime}(1)}
\end{array}\right], } \\
l_{i_{1} i_{2}}^{j_{1}^{\prime}}= & {\left[\begin{array}{ccc}
l_{\alpha_{1} \alpha_{2}}^{\beta_{1}^{\prime}} & l_{\alpha_{1} \alpha_{2}}^{\beta_{1}^{\prime}} & 0 \\
l_{\alpha_{1} \alpha_{2}(1)}^{\beta_{1}^{\prime}} & l_{\alpha_{1} \alpha_{2}(1)}^{\beta_{1}^{\prime}(1)} & 0 \\
l_{\alpha_{1} \alpha_{2}(2)}^{\beta_{1}^{\prime}} & l_{\alpha_{1} \alpha_{2}(2)}^{\beta_{1}^{\prime}(1)} & 0 \\
0 & l_{\alpha_{1}(1) \alpha_{2}(1)}^{\beta_{1}^{\prime}(1)} & 0 \\
l_{\alpha_{1}(1) \alpha_{2}(2)}^{\beta_{1}^{\prime}} & l_{\alpha_{1}(1) \alpha_{2}(2)}^{\beta_{1}^{\prime}(1)} & 0 \\
0 & l_{\alpha_{1}(2) \alpha_{2}(2)}^{\beta_{1}^{\prime}(1)} & l_{\alpha_{1}(1) \alpha_{2}(1)}^{\beta_{1}^{\prime}(1)}
\end{array}\right] . }
\end{aligned}
$$

The above structure is also a generalisation of the $(\text { a.t. })^{2}$-structure. 


\section{Compatible $G_{2}^{1}$ and $(a . p .)_{R}^{2}$ structures}

An $n$-dimensional differentiable manifold $V_{n}$ of class $C^{\infty}$ admitting a $G_{1}$-structure of rank $p$, with $n \geq 2 p$, can also have ([1]) a structural group consisting of matrices of the form,

$$
\begin{aligned}
& {\left[\begin{array}{ccc}
T & S & 0 \\
0 & K & 0 \\
P & N & K
\end{array}\right], \quad T \in L_{n-2 p}, S \in \operatorname{Hom}\left(R^{p}, R^{n-2 p}\right),} \\
& K \in L_{p}, N \in \operatorname{End}\left(R^{p}, R^{p}\right), P \in \operatorname{Hom}\left(R^{n-2 p}, R^{p}\right) .
\end{aligned}
$$

Then, the tensor $J$ will be represented by the matrix,

$$
\left[\begin{array}{lll}
0 & 0 & 0 \\
0 & 0 & 0 \\
0 & I & 0
\end{array}\right] .
$$

The $G_{1}$-adapted basis is of the form,

$$
\left\{e_{\alpha}, e_{\alpha(1)}, e_{\alpha(2)}\right\}, \quad \alpha=1,2, \ldots, n-2 p, \alpha(1), \alpha(2)=1,2, \ldots, p
$$

where $\left\{e_{\alpha}, e_{\alpha(2)}\right\}$ and $\left\{e_{\alpha(1)}\right\}$ define a basis of Ker $J_{x}$ and $S_{x}$, respectively.

Similarly, the $G_{1}^{2}$-structure of rank $p+\left(\begin{array}{c}p+1 \\ 2\end{array}\right)$ and $n+\left(\begin{array}{c}n+1 \\ 2\end{array}\right) \geq$ $2\left(p+\left(\begin{array}{c}p+1 \\ 2\end{array}\right)\right)$, can have a structural group $G_{1}^{2}$ with matrices of the form given by (3.6) where,

$$
\begin{aligned}
l_{i_{1}}^{j_{1}^{\prime}}= & {\left[\begin{array}{ccc}
l_{\alpha_{1}}^{\beta_{1}^{\prime}} & l_{\alpha_{1}}^{\beta_{1}^{\prime}(1)} & 0 \\
0 & l_{\alpha_{1}(1)}^{\beta_{1}^{\prime}(1)} & 0 \\
l_{\alpha_{1}(2)}^{\beta_{1}^{\prime}} & l_{\alpha_{1}(2)}^{\beta_{1}^{\prime}(1)} & l_{\alpha_{1}(1)}^{\beta_{1}^{\prime}(1)}
\end{array}\right], } \\
l_{i_{1} i_{2}}^{j_{1}^{\prime}}= & {\left[\begin{array}{ccc}
l_{\alpha_{1} \alpha_{2}}^{\beta_{1}^{\prime}} & l_{\alpha_{1} \alpha_{2}}^{\beta_{1}^{\prime}(1)} & 0 \\
l_{\alpha_{1} \alpha_{2}(1)}^{\beta_{1}^{\prime}} & l_{\alpha_{1} \alpha_{2}(1)}^{\beta_{1}^{\prime}(1)} & 0 \\
l_{\alpha_{1} \alpha_{2}(2)}^{\beta_{1}^{\prime}} & l_{\alpha_{1} \alpha_{2}(2)}^{\beta_{1}^{\prime}(1)} & 0 \\
0 & l_{\alpha_{1}(1) \alpha_{2}(1)}^{\beta_{1}^{\prime}(1)} & 0 \\
l_{\alpha_{1}(1) \alpha_{2}(2)}^{\beta_{1}^{\prime}} & l_{\alpha_{1}(1) \alpha_{2}(2)}^{\beta_{1}^{\prime}(1)} & 0 \\
l_{\alpha_{1}(2) \alpha_{2}(2)}^{\beta_{1}^{\prime}} & l_{\alpha_{1}(2) \alpha_{2}(2)}^{\beta_{1}^{\prime}(1)} & l_{\alpha_{1}(1) \alpha_{2}(1)}^{\beta_{1}^{\prime}(1)}
\end{array}\right] . }
\end{aligned}
$$


The tensor $J$ can be represented by the matrix,

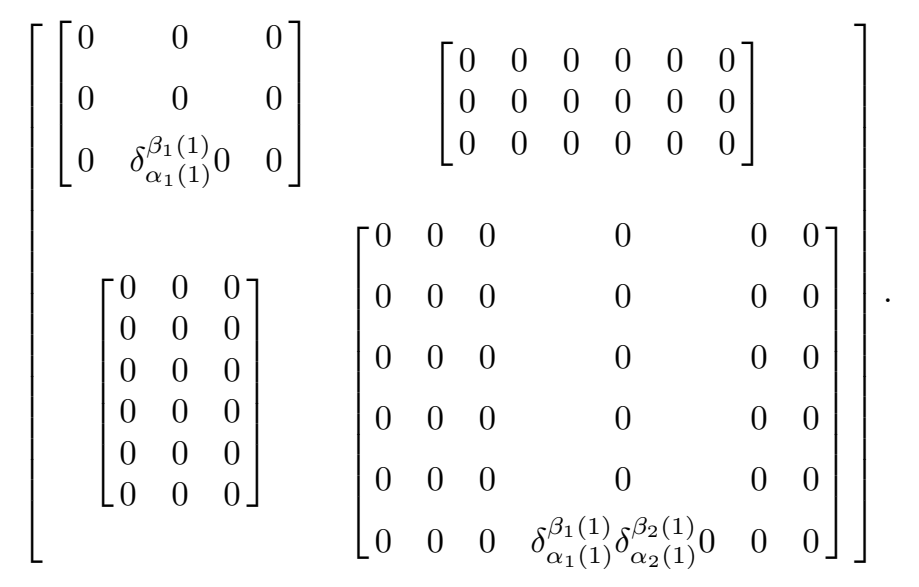

The $G_{1}^{2}$-adapted basis is of the form

$$
\begin{array}{r}
\left\{\left(e_{\alpha_{1}}, e_{\alpha_{1}(1)}, e_{\alpha_{1}(2)}\right),\left(e_{\alpha_{1} \alpha_{2}}, e_{\alpha_{1} \alpha_{2}(1)}, e_{\alpha_{1} \alpha_{2}(2)}\right.\right. \\
\left.\left.e_{\alpha_{1}(1) \alpha_{2}(1)}, e_{\alpha_{1}(1) \alpha_{2}(2)}, e_{\alpha_{1}(2) \alpha_{2}(2)}\right)\right\} \\
\alpha=1,2, \ldots, n-2 p, \quad \alpha(1), \alpha(2)=1,2, \ldots, p
\end{array}
$$

where $\quad\left\{\left(e_{\alpha_{1}}, e_{\alpha_{1}(1)}\right),\left(e_{\alpha_{1} \alpha_{2}}, e_{\alpha_{1} \alpha_{2}(1)}, e_{\alpha_{1} \alpha_{2}(2)}, e_{\alpha_{1}(1) \alpha_{2}(1)}, e_{\alpha_{1}(1) \alpha_{2}(2)}\right)\right\}$ and $\left\{e_{\alpha_{1}(2)}, e_{\alpha_{1}(2) \alpha_{2}(2)}\right\}$ define a basis of Ker $J_{x}$ and $S_{x}$, respectively.

Now, we assume that the manifold $V_{n}$ admits a distribution $\Delta$ of the second order tangent space $T_{x}^{2}\left(V_{n}\right)$ of dimension $n+\left(\begin{array}{c}n+1 \\ 2\end{array}\right)-$ $2\left(p+\left(\begin{array}{c}p+1 \\ 2\end{array}\right)\right)$.

Definition 4.1. A distribution $\Delta$ is compatible with the $G_{1}^{2}$-structure defined by the tensor $J$, if $\Delta$ is supplementary of $J\left(T_{x}^{2}\left(V_{n}\right)\right)$ in the $\operatorname{Ker} J_{x}$.

Comparing the matrices (3.7) and (4.1) we have:

Proposition 4.1. The differentiable manifold $V_{n}$ admits a distribution $\Delta$ compatible with the $G_{1}^{2}$-structure, if and only if, $V_{n}$ is equiped with an (a.tr. $)^{2}$-structure. 
Definition 4.2. An almost product structure of second order is compatible with the generalised almost tangent structure of second order, if

i) Ker $J_{x}$ contains $\Delta$ and

ii) $J_{x}$ applies the $p+\left(\begin{array}{c}p+1 \\ 2\end{array}\right)$ second order base vectors of the complement space of $\Delta$, on the rest, not in $\Delta, p+\left(\begin{array}{c}p+1 \\ 2\end{array}\right)$ base vectors of $\operatorname{Ker} J_{x}$.

Proposition 2. A necessary and sufficient condition, that an $(\text { a.p. })_{R^{-}}^{2}$ structure of dimension $n+\left(\begin{array}{c}n+1 \\ 2\end{array}\right)-2\left(p+\left(\begin{array}{c}p+1 \\ 2\end{array}\right)\right)$, with $2 p+q=$ $n$ is compatible with the $G_{1}^{2}$-structure of rank $p+\left(\begin{array}{c}p+1 \\ 2\end{array}\right)$, is the $n$ dimensional differentiable manifold $V_{n}$ of class $C^{\infty}$ to admit a structure defined by matrices of the form (3.6) with,

$$
\begin{aligned}
l_{i_{1}}^{j_{1}^{\prime}}= & {\left[\begin{array}{ccc}
l_{\alpha_{1}}^{\beta_{1}^{\prime}} & 0 & 0 \\
0 & l_{\alpha_{1}(1)}^{\beta_{1}^{\prime}(1)} & 0 \\
0 & l_{\alpha_{1}(2)}^{\beta_{1}^{\prime}(1)} & l_{\alpha_{1}(1)}^{\beta_{1}^{\prime}(1)}
\end{array}\right], } \\
l_{i_{1} i_{2}}^{j_{1}^{\prime}}= & {\left[\begin{array}{ccc}
l_{\alpha_{1} \alpha_{2}}^{\beta_{1}^{\prime}} & 0 & 0 \\
l_{\alpha_{1} \alpha_{2}(1)}^{\beta_{1}^{\prime}} & 0 & 0 \\
l_{\alpha_{1} \alpha_{2}(2)}^{\beta_{1}^{\prime}} & 0 & 0 \\
0 & l_{\alpha_{1}(1) \alpha_{2}(1)}^{\beta_{1}^{\prime}(1)} & 0 \\
0 & l_{\alpha_{1}(1) \alpha_{2}(2)}^{\beta_{1}^{\prime}(1)} & 0 \\
0 & l_{\alpha_{1}(2) \alpha_{2}(2)}^{\beta_{1}^{\prime}(1)} & l_{\alpha_{1}(1) \alpha_{2}(1)}^{\beta_{1}^{\prime}(1)}
\end{array}\right], } \\
l_{\alpha_{1}}^{\beta_{1}^{\prime}} & {\left[\begin{array}{ccc}
L_{n-2 p}, l_{\alpha_{1}(1)}^{\beta_{1}^{\prime}(1)} \in L_{p}, l_{\alpha_{1}(2)}^{\beta_{1}^{\prime}(1)} \in \operatorname{End}\left(R^{p}, R^{p}\right) .
\end{array}\right.}
\end{aligned}
$$

\section{References}

1. A. Carfagna D'Andrea, Une caractérisation du fibré tangent à un feuilletage, C. R. Acad. Sci. Paris Sér. I Math. 301(3) (1985), 77-80. 
2. A. Carfagna d'Andrea and R. Di Febo Marinelli, Su una generalizzazione delle structure quasi tangenti, Rend. Mat. Appl. (7) 2 (1982), 257-267.

3. B. Cenkl, On the $G$-structures of higher order, Câsopis. Pêst. Mat. 90 (1965), 26-32.

4. R. S. Clark and M. R. Bruckheimer, Sur les structures presque tangentes, C. R. Acad. Sci. Paris Sér. I Math. 251 (1960), 627-629.

5. D. Demetropoulou-Psomopoulou, A prolongation of the real almost product structure of a differentiable manifold, Demonstratio Math. 20(3-4) (1987), 423-439.

6. D. Demetropoulou-Psomopoulou, $G_{1}$-structures of second order, Publ. Mat. 36 (1992), 51-64.

7. C. Ehresmann, Les prolongements d'une variété différentiable. I Calcul des jets, prolongements principal, C. R. Acad. Sci. Paris Sér. I Math. 233 (1951), 598-600.

8. C. Ehresmann, Les prolongements d'une variété différentiable, Cong. Un. Mat. It. Taormina (1951), 317-325.

9. C. Ehresmann, Les prolongements d'une variété différentiable. V Convariants différentiels et prolongements d'une structure infinitesimale, C. R. Acad. Sci. Paris Sér. I Math. 234 (1952), 1424-1425.

10. C. Ehremsann, Introduction à la théorie des structures infinitésimales et des pseudogroupes de Lie, Coll. Inter. du C.N.R.S., Geom. Diff. Strasbourg (1953), 97-100.

11. H. A. Eliopoulos, Structures presque tangents sur les variétés différentiables, C. R. Acad. Sci. Paris Sér. I Math. 255 (1962), 1563-1565.

12. H. A. Eliopoulos, On the connections and the holonomy group of certain G-structures, Ann. Mat. Pura Appl.(4) 78 (1968), 1-12.

13. G. Legrand, Ètude d'une géneralisation des structures presques complexes sur les variétés différentiables, Thése, Paris (1968).

14. J. Lehmann-Lejeune, Sur l'integrabilité de certaines $G$-structures, C. R. Acad. Sci. Paris Sér. I Math. 258 (1964), 5326-5329.

15. M. DE LÉOn And P. R. RodríGuez, "Generalized Classical Mechanics and Field Theory," North-Holland Mathematical Studies 112, North-Holland, Amsterdam, 1985.

16. Tong Van Due, Structures presques transverse, J. Differential Geom. 14 (1979), 215-219. 
17. K. Yano And S. Ishihara, Tangent and Cotangent Bundles, Pure and Appl. Math. Ser. 16, Marcel Dekker, New York, 1973.

\author{
Department of Mathematics \\ Faculty of Sciences \\ University of Thessaloniki \\ 54006 Thessaloniki \\ GREECE
}

Primera versió rebuda el 12 d'Abril de 1996, darrera versió rebuda el 3 de Setembre de 1996 